\title{
Vesico-cervical fistulae: case series
}

\begin{abstract}
Background: Vesico-cervical fistula (VCF) is an extremely rare complication of cesarean section, and only sporadic cases have been reported.

Objectives: We wanted to analyze the incidence, symptomatology, diagnosis and therapeutic aspects of vesico-cervical fistula.

Methods: In a six years period we treated four women with vesico-cervical fistula, mean age 34 years, all following a cesarean section.

Results: Here we present the diagnosis, management and outcome of women with this complication in our institution.

Conclusion: This report should increase awareness of this uncommon complication and aid in prompt and comprehensive evaluation and management.
\end{abstract}

Keywords: genito-urinary, vaginal examination, cystography, uterine cervix, anastomosis
Volume 9 Issue I - 2020

\author{
Masha Ben Zvi,' Matvey Tsivian, ${ }^{2}$ Menahem \\ Neuman, ${ }^{3}$ Ami Sidi, ${ }^{2}$ Alexander Tsivian ${ }^{2}$ \\ 'Department of Obstetrics and Gynecology, School of Medicine, \\ Tel Aviv University, Israel \\ ${ }^{2}$ Department of Urologic Surgery, E. Wolfson Medical Center, \\ Holon, and Sackler School of Medicine, Tel Aviv University, Tel \\ Aviv, Israel \\ ${ }^{3}$ Urogynecology Unit, Dept. of Obstetrics and Gynecology, \\ Faculty of Medicine, Bar-llan University, Israel
}

\author{
Correspondence: Masha Ben Zvi, Department of Obstetrics \\ and Gynecology, EWolfson Medical Center, \\ PO. Box 5, Holon, 58100, Israel, Tel +972-3-502-8653, \\ Email msha.tsivian@gmail.com
}

Received: February 13, 2020 | Published: February 28, 2020

\begin{abstract}
Abbreviations: VCF, Vesico-cervical fistula; CS, caesarean section; CTU, computed tomography urography; VUF, Vesico-uterine fistulae; E. coli, escherichia coli

\section{Introduction}

Vesico-cervical fistulae (VCF) are extremely rare complications of caesarean section (CS). Vesico-uterine fistulae(VUF) represent 1-4\% of genitourinary fistulae, ${ }^{1}$ but only sporadic cases of VCF have been reported. ${ }^{2}$ In light of the increasing prevalence of this complication due to more frequent use of CS increased awareness is warranted. Herein we present 4 cases VCF, their diagnosis, management, treatment and outcome.
\end{abstract}

\section{Case reports}

After local ethical committee approval data of genito-urinary fistulae in our hospital was retrospectively revised. Four patients diagnosed with VCF were identified; they referred to our hospital between 2008 and 2015. Their medical records were reviewed and the time of presentation, prior treatment, diagnosis, management and outcomes were recorded. All four cases followed a low segment CS. Patient ages were 30-40 (mean-34) years.

\section{Case I}

A 30 year-old female suffered a genitourinary fistula a month after CS. ${ }^{3}$ Vaginal examination revealed anterior vaginal wall laceration and uterine cervix rupture. Reconstruction of the injured uterine cervix, the anterior vaginal wall, and the posterior bladder wall were carried out. Nevertheless, trans-vaginal urine leakage persisted, and a vesico-vaginal fistula was suspected. In view of an apparently intact vaginal channel and persistent urinary leakage, intra-vesical dye was instilled, demonstrating urine outflow through the uterine cervix. VCF was identified on retrograde cystography.

Urine culture obtained during hospitalization revealed a multidrug resistant E. coli, suprapubic and urethral catheters were removed and the patient was placed on antibiotics. Four weeks later the patient returned to the clinic reporting spontaneous urination with small amount of urine leakage from the vagina. Urine was sterile at that time. Repeat cystoscopy, intravesical dye test and cystography showed an intact bladder suggesting spontaneous closure of the VCF following 4weeks of conservative management. However, mild urinary leakage persisted; an intra-venous indigo carmine study was performed demonstrating a leak from the uterine cervix channel. An additional fistulous component between the right ureter and uterine cervix was identified on CTU. The patient underwent a ureteral re-implantation with stent placement (removed four weeks postoperatively) and her postoperative course was uneventful. Twelve months after the procedure, full continence was reported with no signs or symptoms of genitourinary fistula and normal ultrasound examination.

\section{Case 2}

A 34 year-old female delivered by CS, during which the anterior vaginal wall and the bladder were damaged; she was re-operated, but three days later urine leakage appeared. The patient was referred to our hospital with a Foley catheter visible through the uterine cervix. A significant vaginal rupture of the anterior wall was seen, which continued to the uterine cervix. Cystoscopy revealed partial absence of the posterior bladder wall, together with part of the anterior vaginal wall and the anterior uterine cervix. A vesico-cervical-vaginal fistulae repair was carried out by an abdominal approach. The postsurgical course was uneventful. A month later pelvic examination was unremarkable, the catheter was removed and the patient passed urine spontaneously. Retrograde cystography was normal.

\section{Case 3}

A 33 years-old female known to have a congenital single kidney, was admitted to our department 30 months following a low segment CS. Patient's history was remarkable for distressing long lasting urinary incontinence in the first few months following the CS, that with time transformed to urinary incontinence only with effort and during the night. The menstrual flow was normal. Numerous 
diagnostic attempts failed to verify the etiology of the urinary leakage. A thorough investigation at our institution, including pelvic examination, cystoscopy, cystography and CTU, showed a fistula communicating the bladder to the uterine cervix with no involvement of the upper urinary tract (Fig.1), the patient underwent fistula repair by abdominal approach with cervical reconstruction. During the surgery, after separating the bladder from the cervix, the cervix appeared to be nearly divided in two. The question of whether to perform a hysterectomy or to continue with cervix reconstruction was raised. Due to the age of the patient and her reproductive plan we decided to perform a reconstruction with end to end anastomosis leaving Nelaton catheter in site. Postoperative course was uneventful. On follow up, full continence was reported with no signs or symptoms of vesico-cervical fistula. The menstruation flow was normal.

\section{Case 4}

A 40 year-old female was referred to our hospital eight months following her fifth CS, combined with supracervical hysterectomy because of placenta accreta and massive intrapartum bleeding. Bladder injury was identified and repaired at surgery. Continuous urinary incontinence, urgency and frequency with recurrent urinary tract infections occurred in the puerperium. Vesico-vaginal fistula was diagnosed on cystoscopy and retrograde cystography. The patient underwent fistula repair by vaginal approach. During the surgery while separating the fistulous tract we were surprised to discover the uterine cervix stump, we noticed that the cervix was involved in the fistulous pathway (Fig. 2), it prompted additional technical difficulties. Postoperative course was uneventful. On control cystography no communication evidence was observed with resolution of the fistula and the symptoms. Urinary incontinence was the presenting symptom in all four cases. All patients had vaginal examination, cystoscopy and cystography. In two cases computed tomography urography (CTU) was necessary to confirm the diagnosis. All fistulae were successfully treated and full continence was reported at minimal six months follow up with no clinical or imaging signs of recurrence.

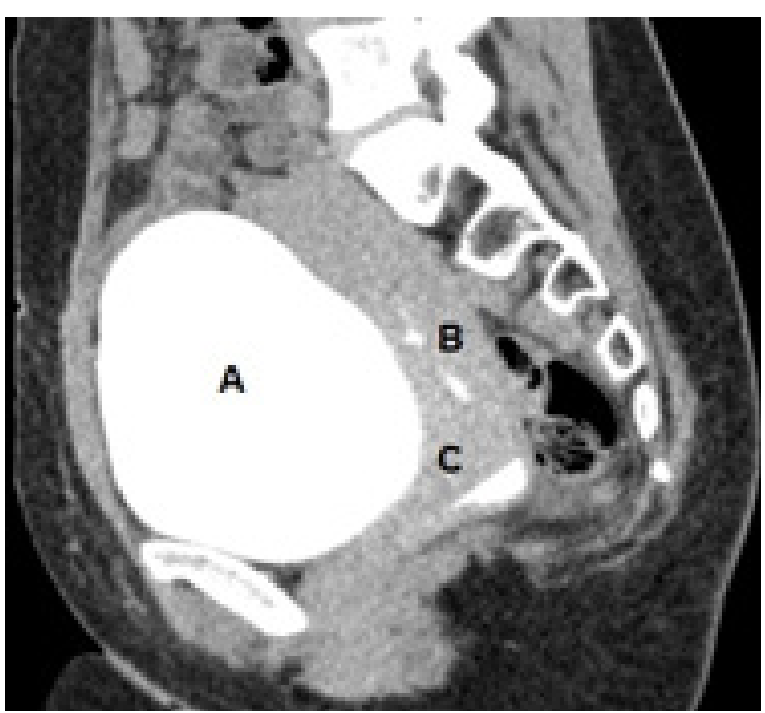

Figure I CT Cystography
A- Contrast material in the bladder
B- Contrast material in the uterine cavity and the cervical canal
C- Contrast material in the vagina

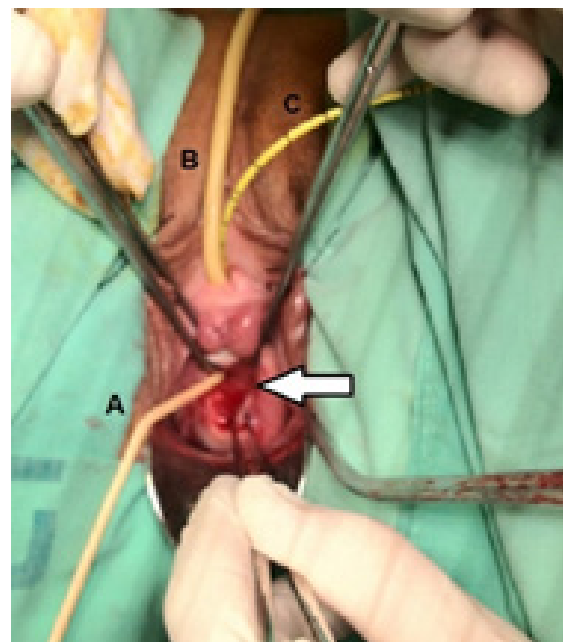

Figure 2 Fistulous tract
A- Catheter in the fistulous tract
B- Urethral catheter
C- Urethral catheter

Cervical canal indicated by arrow

\section{Discussion}

Vesico-cervical fistula is a rare complication of CS, with few reports published, ${ }^{2-4}$ and in some cases it might be misdiagnosed as vesico-uterine fistula. We believe it is important to sub-classify the vesico-cervical fistula as a separate entity, since the mechanism of its formation, its presentation and the surgical repair differ significantly from those of vesico-uterine and vesico-vaginal fistulae. In the four cases of VCF we have presented CS was the primary etiology. ${ }^{2}$, 3 Vesico-cervical fistulae may present either immediately after a $\mathrm{CS}$ or sometime later. Even though all four cases were immediate complications of CS, the third and the fourth cases were diagnosed later.

In VCF urinary incontinence presents early in the post-operative period and may be total, intermittent, continuous or transient. ${ }^{5}$ Other symptoms may include menouria: ${ }^{5,6}$ when the fistula opens above the internal cervical os (vesico-uterine fistula), the menstrual blood flow may be forced to enter the bladder due to the functional sphincter mechanism and the pressure difference between the bladder and the uterus (up to $50 \mathrm{~cm} \mathrm{H}_{2} \mathrm{O}$ during voiding phase), especially during the menstrual cycle phase, when it can reach $130-160 \mathrm{~cm} \mathrm{H}_{2} \mathrm{O}$. On the other hand, continuous urinary leakage occurs when the fistula opens below the internal cervical os, ${ }^{7}$ because there is no sphincter mechanism. Thus, normal menses with persisting urinary leakage, might suggest VCF. The diagnosis should be confirmed with a pelvic examination, imaging techniques such as cystoscopy, cystography and CTU. With the help of these techniques we were able to diagnose all four cases, including the combined fistula described in the first case.

Treatment for vesico-cervical and vesico-uterine fistulae following caesarean section may be conservative or surgical. ${ }^{1}$ Conservative management entails the insertion of an indwelling catheter for 2-3 weeks. This approach has been reported to be successful in $5 \%$ of vesico-uterine fistulae, but is controversial in the case of VCF. $2,4,8$ 
Surgical management is still the preferred therapy for VCF. For patients desiring preservation of fertility, uterine-sparing surgery is to be considered. ${ }^{9}$ Our approach to urogenital fistulae is aggressive, ${ }^{10}$ and immediate reconstructive surgery is considered. In VCFs, however, our experience suggests that a conservative approach may be considered since one of the four cases resolved with conservative trial. Long-standing fistulae are unlikely to resolve with a non-surgical approach. All four cases presented here were different regarding presentation, diagnosis and treatment. It is important to emphasize that even in a small sample of cases the variability of presentation, diagnosis and treatment is very high. Therefore it is of importance to remember the possibility of encountering this entity and to be ready to treat it accordingly.

\section{Conclusion}

This report should raise awareness to these uncommon complications and aid in prompt and comprehensive evaluation.

\section{Acknowledgments}

None.

\section{Conflicts of interest}

The author declares that there are no conflicts of interest.

\section{Funding}

None.

\section{References}

1. Yip SK, Leung TY. Vesicouterine fistulae: an updated review. Int Urogynecol J Pelvic Floor Dysfunct. 1998;9:252-256.
2. Mahmoud S, Arunkalaivanan AS, Devarajan R, et al. Vesicocervical fistula - a rare complication secondary to caesarean secion. Int Urogynecol J Pelvic Floor Dysfunct. 2004;15(6):439-441.

3. Tsivian M Jr, Tsivian M, Sidi AA, et al. Uretero-vesico-cervical fistula following a caesarean section: a unique case report. Int Urogynecol J. 2012;23(11):1639-1641.

4. Golomb J, Ben Chaim J, Goldwasser B, et al. Conservative treatment of a vesicocervical fistula resulting from Shridokar cervical cerclage. $J$ Urol. $1993 ; 149(4): 833-834$.

5. Jozwik M, Jozwik M. Clinical classification of vesicouterine fistula. Int J Gynaecol Obstet. 2000;70(3):353-357.

6. Youssef AF. Menouria following lower segment cesarean section; a syndrome. Am J Obstet Gynecol. 1957;73(4):759-767.

7. Kihl B, Nilson AE, Pettersson S. Postcesarean vesico-uterine fistula. Acta Obstet Gynecol Scand. 1980;59(3):277-280.

8. Varawalla NY, Krishna UR. Conservative management of traumatic vesico-cervical fistula. J Post-grad Med. 1987;33(2):102-104.

9. Di Marco CS, Di Marco DS, Klingele CJ, et al. Vesicouterine fistula: a review of eight cases. Int Urogynecol J Pelvic Floor Dysfunct. 2006;17(4):395-399.

10. Tsivian A, Uchvatkin G, Shtricker A, et al. In support of early treatment of postoperative vesicovaginal fistulas. J Pelv Med \& Surg. 2006;12(4):197200 . 